\title{
Night-to-night variation of pulse oximetry in children with sleep-disordered breathing
}

\author{
Rachael M Burke, ${ }^{1,2}$ Barbara Maxwell, ${ }^{2}$ Carolyn Hunter, ${ }^{2}$ David Graham, ${ }^{2}$ \\ Dara O'Donoghue, ${ }^{2}$ Michael D Shields ${ }^{1,2}$
}

- Additional material is published online only. To view please visit the journal online (http://dx.doi.org/10.1136/ archdischild-2015-308981).

${ }^{1}$ Centre for Infection and Immunity, Queen's University Belfast, Belfast, UK

${ }^{2}$ Royal Belfast Hospital for Sick Children, Belfast Health \& Social Care Trust, Belfast, UK

\section{Correspondence to}

Prof Michael D Shields, Centre for Infection and Immunology, Health Sciences Building, 97 Lisburn Road, Belfast BT9 7AE, UK; m.shields@qub.ac.uk

Received 14 May 2015 Revised 15 February 2016 Accepted 21 February 2016 Published Online First 11 March 2016

\section{SLinked}

- http://dx.doi.org/10.1136/ archdischild-2016-310483

\section{CrossMark}

To cite: Burke RM,

Maxwell B, Hunter C, et al. Arch Dis Child

2016;101:1095-1099.

\section{ABSTRACT}

Background Sleep-disordered breathing is a common and serious feature of many paediatric conditions and is particularly a problem in children with Down syndrome. Overnight pulse oximetry is recommended as an initial screening test, but it is unclear how overnight oximetry results should be interpreted and how many nights should be recorded.

Methods This retrospective observational study evaluated night-to-night variation using statistical measures of repeatability for 214 children referred to a paediatric respiratory clinic, who required overnight oximetry measurements. This included 30 children with Down syndrome. We measured length of adequate trace, basal $\mathrm{SpO}_{2}$, number of desaturations ( $>4 \% \mathrm{SpO}_{2}$ drop for $>10 \mathrm{~s}$ ) per hour ('adjusted index') and time with $\mathrm{SpO}_{2}<90 \%$. We classified oximetry traces into normal or abnormal based on physiology.

Results 132 out of $214(62 \%)$ children had three technically adequate nights' oximetry, including 13 out of $30(43 \%)$ children with Down syndrome. Intraclass correlation coefficient for adjusted index was 0.54 (95\% $\mathrm{Cl} 0.20$ to 0.81 ) among children with Down syndrome and $0.88(95 \% \mathrm{Cl} 0.84$ to 0.91$)$ for children with other diagnoses. Negative predictor value of a negative first night predicting two subsequent negative nights was 0.2 in children with Down syndrome and 0.55 in children with other diagnoses.

Conclusions There is substantial night-to-night variation in overnight oximetry readings among children in all clinical groups undergoing overnight oximetry. This is a more pronounced problem in children with Down syndrome. Increasing the number of attempted nights' recording from one to three provides useful additional clinical information.

\section{INTRODUCTION}

Overnight pulse oximetry is commonly used as a screening tool for sleep-disordered breathing in children. Formal polysomnography is the gold standard to diagnose sleep-disordered breathing, ${ }^{1}$ but this is not often available as a first-line investigation due to patient and parent burden, need for inpatient admission and cost. For example, a survey in 2005 identified only 10 paediatric sleep laboratory beds in the whole of the UK. ${ }^{2}$ Overnight pulse oximetry is advantageous as it is readily available, does not require admission to hospital overnight and has minimal upheaval for children and parents. Although it is recommended as a first-line screening test only, for many children overnight pulse oximetry is the only diagnostic test available to identify sleep-disordered breathing due to the difficulties of

\section{What is already known on this topic}

- Overnight polysomnography is the gold standard to diagnose sleep-disordered breathing in children, but is difficult to access routinely.

- Overnight pulse oximetry is recommended for screening of sleep-disordered breathing, but there is little evidence about how best to interpret overnight oximetry results.

- Children with Down syndrome should be tested for sleep-disordered breathing annually up to age 6 years.

\section{What this study adds}

- There is substantial within-patient night-to-night variation when recording sequential nights of overnight oximetry.

- Many children investigated for suspected sleep-disordered breathing (39 out of 84 in this study) with one normal night of oximetry go on to have abnormal oximetry in sequential subsequent nights.

accessing polysomnography. ${ }^{2-4}$ There is no clear research data on the levels of overnight oximetry abnormality that predict clinical benefit from intervention, although best practice guidelines have been published. ${ }^{1} 2556$ Additionally, some small studies have shown night-to-night variation in sleep studies, ${ }^{78}$ but it is unclear how significant this variation is in clinical practice. This a clinically important area as UK guidelines from Working Party on Sleep Physiology and Respiratory Control Disorders in Childhood (SPARDIC) endorsed by the Royal College of Paediatrics and Child Health $(\mathrm{RCPCH})$ recommend annual screening for obstructive sleep apnoea (OSA) for all children with Down syndrome (DS) up to 6 years of age $^{2}$ due to the high risk of developing pulmonary hypertension in this group. The Down Syndrome Medical Interest Group (also endorsed by the $\mathrm{RCPCH}$ ) recommend a symptom-based screen with referral for further testing if positive. ${ }^{9}$ If the SPARDIC guideline were to be fully implemented, a very large number of children would undergo overnight oximetry, with the attendant difficulties in interpreting results. It is not likely to be feasible 
in the near future for formal polysomnography to be available to all children with DS annually; therefore, it is vitally important to be able to best understand the strengths, limitations and repeatability of overnight oximetry.

At the Royal Belfast Hospital for Sick Children, our clinical practice has been to record three consecutive nights of overnight oximetry readings when screening for sleep-disordered breathing. This practice was instituted by the senior author (MDS) who, when first setting up home oximetry in Northern Ireland many years ago, had observed marked night-to-night variability especially in DS. The present study aims to assess whether recording three nights provides additional useful data or whether recording only one night of oximetry would be adequate. Specifically we assess (1) does recording three nights' data increase the chance of having a technically sufficient and interpretable night's recording? (2) how repeatable are results night to night? (3) how clinically significant are night-to-night variations in overnight oximetry recordings (ie, does variation lead to a change in clinical classification)?

Children with DS are known to often be restless sleepers, ${ }^{10}$ and we hypothesised that they would have more night-to-night variability compared with other clinical groups and would thus require oximetry over three nights.

\section{METHODS}

This was a retrospective observational study designed as a service development and quality improvement project. Overnight oximetry recordings were taken only when clinically indicated as part of routine practice. Children were classified according to diagnostic category by their clinician at the time of oximetry recording into one of the following categories: DS, neuromuscular disease (such as Duchenne's muscular dystrophy) (NMD), central nervous system (CNS) disorders (such as cerebral palsy), craniofacial structural abnormalities (such as achondroplasia or Pierre Robin syndrome) (structural) and other children referred with suspected OSA with no other diagnosis (OSA). Anonymised data only were collected by researchers.

Overnight pulse oximetry was recorded by means of a Nonin 9600 Avant Digital Pulse Oximeter (Nonin Medical, Plymouth, Minnesota, USA) and Nellcor neonatal-adult $\mathrm{SpO}_{2}$ sensor. Parents were instructed how to use the pulse oximeter and took the device home. All pulse oximetry readings were recorded by parents at home in the child's normal surroundings. The sensor was attached to either the child's finger or big toe. After three nights, parents returned the oximeter and data were downloaded and analysed using nVision software (Nonin Medical).

Length of time of adequate artefact free reading (hours), basal oxygen saturations (defined as average of all $\mathrm{SpO}_{2}$ readings not included in a desaturation event) $\left(\% \mathrm{SpO}_{2}\right)$, length of time with oxygen saturation $<90 \%$ (expressed as a percentage of length of recording) and average number of desaturations per hour (defined as $>4 \%$ drop in $\mathrm{SpO}_{2}$ for $>10$ s, the 'adjusted index') were extracted from the Nonin nVision software and into the anonymised study database. $\mathrm{SpO}_{2}$ was averaged over $4 \mathrm{~s}$ increments.

A technically adequate reading was defined as one with $\geq 4 \mathrm{~h}$ of suitable oximetry recording. A normal pulse oximetry reading was defined as basal $\mathrm{SpO}_{2} \geq 94 \%$; $<2 \%$ of total time with $\mathrm{SpO}_{2}<90 \%$; and adjusted index $<5$. An oximetry recording with abnormalities in any of these perimeters was defined as abnormal. These criteria for normal readings follow those recommended by the $\mathrm{RCPCH}^{2}$ and are in line with published normal values for children. ${ }^{11}$
Data were analysed in Stata 11 (Stata Statistical Software: Release 11, StataCorp 2009, College Station, Texas) and StatsDirect V.3 (StatsDirect statistical software 2013, http://www. statsdirect.com). Bland-Altman plots were used to graphically display agreement of basal $\mathrm{SpO}_{2}$, adjusted index and time with $\mathrm{SpO}_{2}$ below $90 \%$ between nights. The 95\% limits of agreement ${ }^{12}$ and two-way single measures intraclass correlation coefficients (ICCs) were calculated. For pairwise comparisons of continuous variables across clinical groupings, Kruskal-Wallis (non-parametric) tests were used. For comparisons of discrete variables, a $\chi^{2}$ test was used. A Fleiss' $\kappa$ value was calculated for agreement of results across all three nights. Following convention, we defined a $\kappa$ value of $>0.6$ as substantial agreement. ${ }^{13}$

\section{RESULTS}

In total, 214 children had overnight home pulse oximetry recordings between December 2010 and July 2014 arranged through the Royal Belfast Hospital for Sick Children. The average age of children was 4.2 years old (range 2 months to 18.3 years). The majority of the group were otherwise well children with signs and symptoms leading to suspicion of OSA (OSA group) (114 out of 214 (53\%), median age 3.5 years), and this group was used as a baseline group for comparison. There were $23(11 \%)$ children with NMDs (NMD group) (median age 12.3 years), 30 with DS (DS group) (median age 4.8), 26 (12\%) with CNS disorders (CNS group) (median age 3.1 years) and 21 (10\%) with structural craniofacial abnormalities (structural group) (median age 4.4 years). Children with NMD were older than children in the OSA group (12.3 vs 3.5 years, $\mathrm{p}<0.01)$.

The mean length of time of adequate recording was $8.4 \mathrm{~h}$ (SD $2.9 \mathrm{~h}$ ). Regarding the technical adequacy of recording usable results, 25 of our $214(12 \%)$ children did not have sufficient data recorded on the first night to make any assessment of sleep ventilation. Of these, $18(8 \%)$ children went on to record adequate readings on subsequent nights. Also, 132/214 (62\%) children had adequate recordings on all three nights. Seven children (one DS, one CNS, five OSA) had no usable data recorded on any of the three nights. No children (0/23) with NMD had unusable oximetry recordings on night 1 , whereas $6 / 30(20 \%)$ children with DS had an inadequate reading on night 1 . This difference was not statistically significant $(p=0.16)$. Also, 19/ $122(16 \%)$ children under age 5 had unusable readings on night 1 compared with $7 / 91$ (8\%) of children 5 and older. This difference was not statistically significant $(\mathrm{p}=0.08)$.

There was marked night-to-night variation in the oximetry traces of many of the children over the three nights. Table 1 summarises the within-patient difference, SD of differences and 95\% limits of agreement for three nights' pairwise comparisons. Figure 1 shows example Bland-Altman plots of agreement between basal $\mathrm{SpO}_{2}$, adjusted index and time with $\mathrm{SpO}_{2}<90 \%$ in night 1 and night 2 (see e-Figure 1 for comparisons of nights 1 and 3, and 2 and 3). Table 2 shows the ICC across all three nights by clinical condition. The ICC tended to be lower for children with DS; this difference was significant when considering the night-to-night variability of adjusted index.

As described above, overnight oximetry readings were categorised to be either normal or abnormal based on the physiology observed. Overall, 117 out of 207 (57\%) were normal on the first adequate night, and 73 out of 109 (67\%) children with suspected OSA only and no other clinical diagnosis had normal oximetry. This was similar to the proportion of children with NMD, in whom 14 out of 23 (61\%) had normal oximetry $(p=0.58)$. Children with other diagnoses were less likely to have a normal first night; 9 out of 25 (36\%) children with CNS 
Table 1 Mean value for each night (SD), mean within-patient difference, SD of with-patient differences and 95\% limits of agreement across three pairs of nights for each of the three values tested

\begin{tabular}{|c|c|c|c|c|c|}
\hline & $\mathrm{N}$ & Means (SD) & Mean difference & SD of differences & $\begin{array}{l}\text { Bland-Altman } 95 \% \\
\text { limits of agreement }\end{array}$ \\
\hline \multicolumn{6}{|c|}{ Basal oxygen $\mathrm{SpO}_{2}(\%)$} \\
\hline Night 1-night 2 & 179 & $\begin{array}{l}\text { Night } 1=96.1(2.79) \\
\text { Night } 2=96.2(2.47)\end{array}$ & 0.076 & 2.41 & -4.6 to 4.8 \\
\hline Night 1-night 3 & 147 & $\begin{array}{l}\text { Night 1=96.4 (2.3) } \\
\text { Night 3=96.7 (2.02) }\end{array}$ & 0.28 & 1.78 & -3.2 to 3.7 \\
\hline Night 2-night 3 & 150 & $\begin{array}{l}\text { Night 2=96.4 (2.28) } \\
\text { Night 3=96.6 (2.01) }\end{array}$ & 0.15 & 1.45 & -2.7 to 3.0 \\
\hline \multicolumn{6}{|l|}{ Adjusted index } \\
\hline Night 1-night 2 & $178^{*}$ & $\begin{array}{l}\text { Night 1=5.44 (7.30) } \\
\text { Night 2=6.21 (8.76) }\end{array}$ & -0.76 & 4.91 & -8.8 to 10.4 \\
\hline Night 1-night 3 & 147 & $\begin{array}{l}\text { Night } 1=5.21(7.58) \\
\text { Night } 3=5.04(8.00)\end{array}$ & 0.17 & 4.80 & -9.6 to 9.3 \\
\hline Night 2-night $3^{*}$ & $149^{*}$ & $\begin{array}{l}\text { Night 2=5.92 (8.79) } \\
\text { Night 3=5.29 (8.00) }\end{array}$ & 0.63 & 4.45 & -9.4 to 8.1 \\
\hline \multicolumn{6}{|c|}{ Time with $\mathrm{SpO}_{2}<90 \%$ (as \% of time recorded) } \\
\hline Night 1-night 2 & 179 & $\begin{array}{l}\text { Night } 1=4.92(11.9) \\
\text { Night } 2=4.75(10.9)\end{array}$ & 0.17 & 10.6 & -20.8 to 20.6 \\
\hline Night 1-night 3 & 147 & $\begin{array}{l}\text { Night 1=4.11 (10.7) } \\
\text { Night 3=3.04 (8.77) }\end{array}$ & 1.07 & 9.59 & -19.8 to 17.7 \\
\hline Night 2-night 3 & 150 & $\begin{array}{l}\text { Night 2=3.66 (8.86) } \\
\text { Night 3=3.12 (8.76) }\end{array}$ & 0.53 & 6.56 & -13.4 to 12.3 \\
\hline
\end{tabular}

${ }^{*}$ One patient had no data of desaturations per hour recorded on night 2 .

disorders $(\mathrm{p}<0.01), 9$ out of $21(43 \%)$ children with structural abnormalities $(p=0.04)$ and 12 out of $30(41 \%)$ children with DS $(p=0.1)$ had normal first-night oximetry tracings.

There was only moderate concordance of results from oximetry reading on consecutive nights. Restricting the analysis to 132 children with three technically adequate nights' oximetry recording, 84 out of $132(65 \%)$ children had a normal first night's reading. However, nearly half (39 out of $84(46 \%)$ ) of these children had at least one abnormal reading on subsequent nights. Table 3 shows these restricted results broken down by clinical category. If we define a 'true negative' to be a child with three consecutive normal readings, the overall negative predictor value of a first night's normal reading is 0.53 .

Fleiss' $\kappa$ for inter-rater agreement across three nights for children with CNS was $0.63(\mathrm{p}<0.01)$, OSA $0.51(\mathrm{p}<0.01)$, NMD
$0.40 \quad(\mathrm{p}<0.01)$, structural $0.44 \quad(\mathrm{p}<0.01)$ and DS 0.33 $(\mathrm{p}=0.02)$. Overall, Fleiss' $\kappa$ was $0.51(\mathrm{p}<0.01)$.

\section{DISCUSSION}

There is a large amount of night-to-night variability in all children undergoing overnight pulse oximetry, which is especially true of children with DS. Clearly there is a limit to how much oximetry data can be usefully recorded and analysed; we suggest that it may be pragmatic to record three consecutive nights' data rather than a single night's. This will increase the likelihood both of having any usable data and of detecting clinically important abnormalities.

Sleep-disordered breathing is a common paediatric diagnosis, and it is an important feature of a range of paediatric and congenital disorders. Accurately diagnosing sleep-disordered
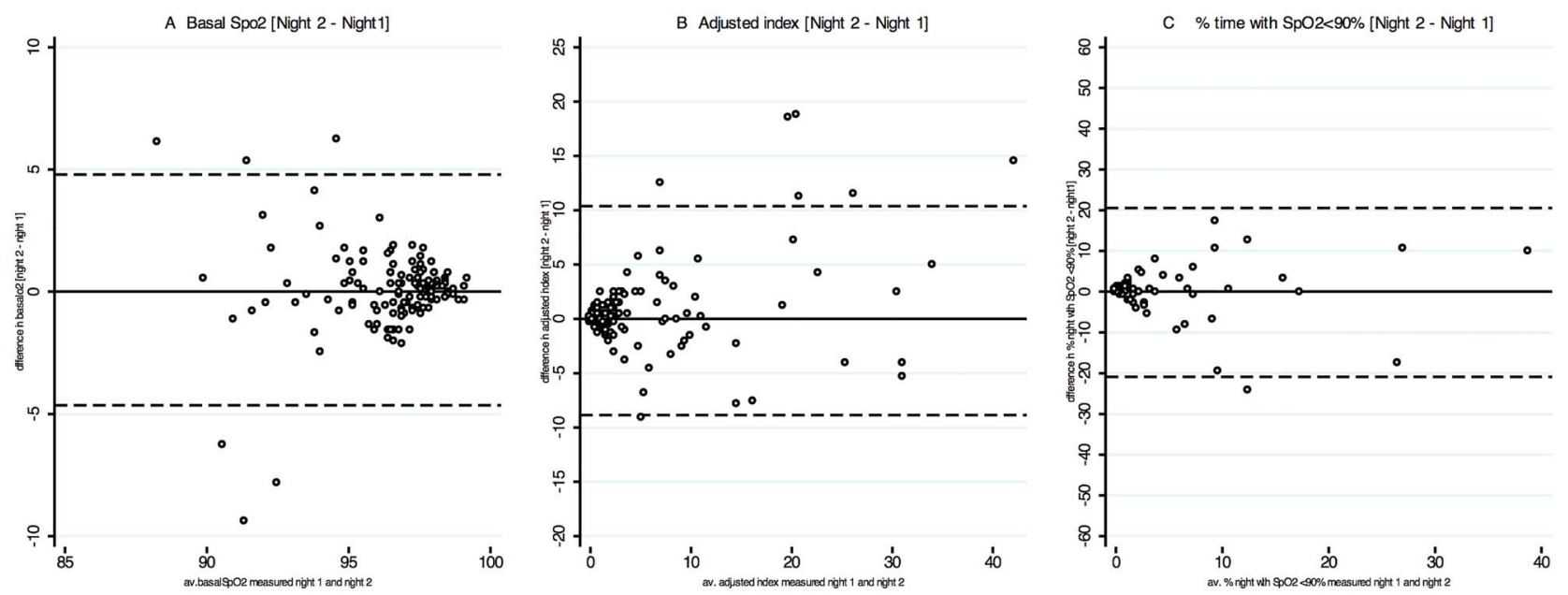

Figure 1 Bland-Altman plots comparing mean versus difference of average $\mathrm{SpO}_{2}(\mathrm{~A})$ adjusted index (B) and time with $\mathrm{SpO}_{2}<90 \%(C)$ in night 1 compared with night 2 . 
Table 2 Intraclass correlation coefficient of measurements over three nights' oximetry recording

\begin{tabular}{llll}
\hline Intraclass correlation coefficient $(95 \% \mathrm{Cl})$ & & & \\
\hline Clinical diagnosis & Basal $\mathrm{SpO}_{2}$ & Adjusted index & Time $\mathrm{SpO}_{2}<90 \%$ \\
\hline Central nervous system disorder, $\mathrm{N}=14$ & $0.74(0.49$ to 0.90$)$ & $0.84(0.66$ to 0.94$)$ & $0.49(0.16$ to 0.77$)$ \\
Suspected obstructive sleep apnoea (no other diagnosis), $\mathrm{N}=69$ & $0.72(0.62$ to 0.81$)$ & $0.90(0.85$ to 0.93$)$ & $0.63(0.51$ to 0.73$)$ \\
Neuromuscular disorder, $\mathrm{N}=20$ & $0.67(0.45$ to 0.84$)$ & $0.57(0.32$ to 0.78$)$ & $0.60(0.35$ to 0.80$)$ \\
Structural craniofacial abnormalities, $\mathrm{N}=15$ & $0.91(0.79$ to 0.96$)$ & $0.94(0.87$ to 0.98$)$ & $0.83(0.66$ to 0.94$)$ \\
Pooled all non-Down syndrome diagnoses, $\mathrm{N}=118$ & $0.74(0.67$ to 0.80$)$ & $0.88(0.84$ to 0.91$)$ & $0.65(0.15$ to 0.78$)$ \\
Down syndrome, $\mathrm{N}=13$ & $0.48(0.15$ to 0.77$)$ & $0.54(0.20$ to 0.81$)$ & $0.48(0.15$ to 0.78$)$ \\
\hline
\end{tabular}

breathing is important so as to allow intervention and prevent development of serious complications such as pulmonary hypertension. Polysomnography is considered to be the gold standard for diagnosis, ${ }^{2}$ but it has significant drawbacks. In addition to cost and access considerations, polysomnography usually measures only one night, thus potentially missing important night-to-night variations, ${ }^{8}$ and takes place in an artificial environment that could artefactually change sleep architecture in children. In addition, access to polysomnography is generally poor; a recent survey of practice in the USA showed that most children who proceeded to surgery for sleep apnoea had never had a polysomnographic diagnosis ${ }^{3}$ and in the UK a survey of paediatrics showed a large unmet need for sleep services and polysomnography. ${ }^{2}$ Overnight pulse oximetry is much more readily available, can be undertaken in a child's normal surroundings (thus likely reducing first-night effect) and is feasible to repeat on consecutive nights in order to capture night-to-night variation. Inherent difficulties with overnight oximetry are that it can be affected by motion artefact and that it measures the whole night regardless of whether the child is awake or asleep.

How best to screen for sleep-disordered breathing is an increasingly important question as best practice guidelines recommend screening all children with NMD (prevalence 1:3000) annually, ${ }^{14}$ in addition to screening all children with signs and symptoms raising suspicion of the diagnosis of OSA. There are varying guidelines regarding DS (prevalence 1:1000), with one guideline recommending testing all children annually up to age $6^{2}$ and another suggesting symptom-based screening. ${ }^{9}$ This highlights that there is currently a wide variation in practice around use of home screening and polysomnography-both in adults and children-and few large studies to provide evidence and guidance.

We found that increasing the overnight oximetry readings from one night to three increases the chance of getting a technically adequate trace that is suitable for interpretation. With one night's recording, $25(12 \%)$ children failed to record adequate data. However, with three nights' recording, only seven (3\%) had no adequate results. There was a trend towards children with DS being less likely to have an adequate trace on the first night of attempted recording, although this was not statistically significant.

We found substantial night-to-night variation in oximetry results. For instance, concerning the adjusted index, the BlandAltman 95\% limits of agreement for difference between night 1 and night 3 in the same patient were -9.6 to +9.3 . Although a proportion of this variation is caused by night-to-night variation in severity among children who had abnormal traces on both

Table 3 Number of children with normal or abnormal overnight oximetry readings on nights 2 and 3, following either normal or abnormal night 1 reading, by clinical group

\begin{tabular}{|c|c|c|c|c|c|}
\hline \multicolumn{6}{|l|}{ Clinical diagnosis } \\
\hline & $\begin{array}{l}\text { 2nd and 3rd night } \\
\text { both normal }\end{array}$ & $\begin{array}{l}\text { 2nd night abnormal, 3rd } \\
\text { night normal }\end{array}$ & $\begin{array}{l}\text { 2nd night normal, 3rd } \\
\text { night abnormal }\end{array}$ & $\begin{array}{l}\text { 2nd and 3rd night both } \\
\text { abnormal }\end{array}$ & Subtotal \\
\hline \multicolumn{6}{|c|}{ First-night oximetry normal and three nights' oximetry recorded ( $N=84$ ) } \\
\hline Central nervous system disorder & 2 & 0 & 1 & 1 & 4 \\
\hline $\begin{array}{l}\text { Suspected obstructive sleep apnoea } \\
\text { (no other diagnosis) }\end{array}$ & 33 & 3 & 7 & 12 & 55 \\
\hline Neuromuscular disorder & 5 & 4 & 1 & 3 & 13 \\
\hline Cranial-facial abnormalities & 3 & 2 & 1 & 0 & 6 \\
\hline Down syndrome & 2 & 1 & 0 & 3 & 6 \\
\hline Total (all diagnoses) & 45 & 10 & 10 & 19 & 84 \\
\hline \multicolumn{6}{|c|}{ First-night oximetry abnormal and three nights' oximetry recorded $(\mathrm{N}=48)$} \\
\hline Central nervous system disorder & 1 & 0 & 0 & 9 & 10 \\
\hline $\begin{array}{l}\text { Suspected obstructive sleep apnoea } \\
\text { (no other diagnosis) }\end{array}$ & 1 & 1 & 0 & 13 & 15 \\
\hline Neuromuscular disorder & 1 & 0 & 0 & 6 & 7 \\
\hline Cranial-facial abnormalities & 0 & 3 & 0 & 6 & 9 \\
\hline Down syndrome & 1 & 1 & 0 & 5 & 7 \\
\hline Total (all diagnoses) & 4 & 5 & 0 & 39 & 48 \\
\hline
\end{tabular}


nights, much of this variation causes change in clinical classification. We found a high proportion of children (46\%) with a normal first-night oximetry, going on to have subsequent abnormal nights. This indicates that recording only one night's data may miss clinically significant oximetry abnormalities. In the absence of access to a gold standard measure (polysomnography) in this cohort, if a 'true negative' is defined as a child with three nights' normal oximetry, then negative predictor value for a normal first night's oximetry trace is only 0.53 , suggesting that relying on only one night's oximetry reading may misclassify children as having no sleep-disordered breathing when they do actually have a clinically significant problem. This fits with previous data on repeatability of oximetry studies, ${ }^{15}$ suggesting that a positive result may be sufficient to make a diagnosis of OSA, but that a negative result cannot rule out the diagnosis and, in this instance, the recommendation has been to move to formal polysomnography. ${ }^{1}$ As with all diagnostic tools with a potential for variable results, it is important that interpretation of oximetry findings is put into the clinical context.

There is limited literature about the repeatability of oximetry measures in polysomnography in the paediatric setting. ${ }^{7}$ One study of 30 children, which excluded patients with DS or any other medical diagnosis, showed an intraclass correlation of 0.86 for apnoeic index and 0.71 for low $\mathrm{SpO}_{2}$ across two nights' polysomnography. ${ }^{7}$ This is similar to our findings for adjusted index and basal $\mathrm{SpO}_{2}$ among the non-DS children in our study. However in that study, in contrast to our study, the observed variation did not lead to reclassification of any of the 30 patients as normal/abnormal across the two nights measured. A larger adult study of 243 patients showed important night-to-night variation, with 28 adults having an abnormal night 2 polysomnograph (defined as $>5$ apnoeic-hypoxic events per hour) following a normal first-night reading. ${ }^{16}$ This suggests that our observed night-to-night variation is in keeping with night-to-night variation seen on polysomnography, and therefore is likely to represent a real phenomenon of within-patient night-to-night fluctuations rather than night-to-night error in oximetry reading.

In view of this clinically important night-to-night variability in overnight oximetry, we suggest that it may be useful to record more than one night's oximetry data. This is especially true in children with DS who had a trend towards more night-to-night variability. Although not formally assessed in this study, the marginal cost and inconvenience to patients and parents of recording three nights rather than one is thought to be minimal, while the extra data recorded may improve clinical management and decision-making, and reduce the risk of missing clinically important abnormalities. While polysomnography remains the gold standard, it is not readily available, and resource limitations mean that is unlikely in the near future to be feasible to substantially increase the number of children undergoing polysomnography in order to meet SPARDIC recommendations to screen all children with DS (or even all symptomatic children) for sleep-disordered breathing annually. ${ }^{2}{ }^{6}$ It would seem prudent to invest more in sleep diagnostics to improve service, and this would be our recommendation in the medium term. However, we suggest that in the interim, pending better availability of alternative modalities, recording three nights rather than one will make overnight oximetry an increasingly useful screening tool. Current guidance is that while a positive result of pulse oximetry can be used to make a diagnosis of OSA, a negative result cannot rule it out and one should proceed to polysomnography and, where this is not available, clinicians must use their judgement while being mindful of the limitations of overnight oximetry. ${ }^{1}$ In the absence of ready access to polysomnography studies, repeating the overnight oximetry may give more confidence in negative result. Newer technology such as mobile phone-based oximetry combined with pulse rate variability has been demonstrated to have good diagnostic accuracy and easily lends itself to multiple nights' testing. ${ }^{4}$ This is an increasingly important area because of the large number of children who are being recommended for screening for sleep-disordered breathing.

Contributors RB preformed the data analysis and was responsible for the first draft of manuscript. BM, CH and DG collected original data and provided comments and revisions to the draft manuscript. $\mathrm{CH}$ also did preliminary data analysis. DOD contributed to the study design and manuscript revisions. MS conceived the study, was responsible for study design and contributed substantially to data analysis and revisions of draft manuscript. All authors had full access to all the data in the study and provided final approval for publication. MS takes responsibility as guarantor of the content of the manuscript.

Competing interests None declared.

Ethics approval No institutional ethical approval was required as there was no change to usual care, and only anonymised data were collected in this service development project.

Provenance and peer review Not commissioned; externally peer reviewed.

\section{REFERENCES}

1 Marcus CL, Brooks LJ, Draper KA, et al. Diagnosis and management of childhood obstructive sleep apnea syndrome. Pediatrics 2012;130:e714-55.

2 Royal College of Paediatrics and Child Health. Working Party on Sleep Physiology and Respiratory Control Disorders in Childhood (SPARDIC). London. 2009.

3 Mitchell RB, Pereira KD, Friedman NR. Sleep-disordered breathing in children: survey of current practice. Laryngoscope 2006;116:956-8.

4 Garde A, Dehkordi P, Karlen W, et al. Development of a screening tool for sleep disordered breathing in children using the phone Oximeter ${ }^{\mathrm{TM}}$. PLOS ONE 2014;9: e112959.

5 Punjabi NM, Aurora RN, Patil SP. Home sleep testing for obstructive sleep apnea: one night is enough! Chest 2013;143:291-4.

6 Hull J, Aniapravan R, Chan E, et al. British Thoracic Society guideline for respiratory management of children with neuromuscular weakness. Thorax 2012;67(Suppl 1): i1-40.

7 Katz ES, Greene MG, Carson KA, et al. Night-to-night variability of polysomnography in children with suspected obstructive sleep apnea. J Pediatr 2002;140:589-94.

8 Scholle S, Scholle HC, Kemper A, et al. First night effect in children and adolescents undergoing polysomnography for sleep-disordered breathing. Clin Neurophysiol 2003; 114:2138-45.

9 Paediatric Service Specification (draft). Services for Children and young People with Down Syndrome. Royal College of Paediatrics and Child Health in assosciation with Down Syndrome Assosciation. 2016. http://www.dsmig.org.uk/wp-content/uploads/ 2015/09/Draft-servcei-spec.pdf

10 Cotton S, Richdale A. Brief report: parental descriptions of sleep problems in children with autism, Down syndrome, and Prader-Willi syndrome. Res Dev Disabil 2006;27:151-61.

11 Urschitz MS, Wolff J, Von Einem V, et al. Reference values for nocturnal home pulse oximetry during sleep in primary school children. Chest 2003;123:96-101.

12 Bland JM, Altman DG. Statistical methods for assessing agreement between two methods of clinical measurement. Lancet 1986;1:307-10.

13 Kottner J, Audigé L, Brorson S, et al. Guidelines for Reporting Reliability and Agreement Studies (GRRAS) were proposed. J Clin Epidemiol 2011;64:96-106.

14 Hull J. British Thoracic Society guideline for respiratory management of children with neuromuscular weakness: commentary. Thorax 2012;67:654-5.

15 Brouillette RT, Morielli A, Leimanis A, et al. Nocturnal pulse oximetry as an abbreviated testing modality for pediatric obstructive sleep apnea. Pediatrics 2000;105:405-12.

16 Le Bon 0, Hoffmann G, Tecco J, et al. Mild to moderate sleep respiratory events: one negative night may not be enough. Chest 2000;118:353-9. 